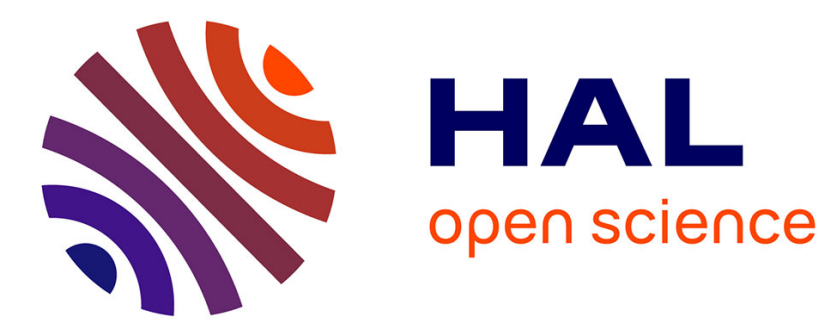

\title{
Nonlinear Fringe-adjusted JTC Based Face Tracking Using an Adaptive Decision Criterion
}

\author{
Isabelle Léonard, Ayman Alfalou, M.S. Alam
}

\section{To cite this version:}

Isabelle Léonard, Ayman Alfalou, M.S. Alam. Nonlinear Fringe-adjusted JTC Based Face Tracking Using an Adaptive Decision Criterion. Optics + Photonics 2011/ SPIE Optical Engineering + Applications, Aug 2011, San Diego, United States. pp.1-6. hal-00607802

\section{HAL Id: hal-00607802 \\ https://hal.science/hal-00607802}

Submitted on 11 Jul 2011

HAL is a multi-disciplinary open access archive for the deposit and dissemination of scientific research documents, whether they are published or not. The documents may come from teaching and research institutions in France or abroad, or from public or private research centers.
L'archive ouverte pluridisciplinaire HAL, est destinée au dépôt et à la diffusion de documents scientifiques de niveau recherche, publiés ou non, émanant des établissements d'enseignement et de recherche français ou étrangers, des laboratoires publics ou privés. 


\title{
Nonlinear Fringe-adjusted JTC Based Face Tracking Using an Adapted Decision Criterion
}

\author{
I. Leonard ${ }^{(1)}$, A. Alfalou $^{(1)}$, M. Alam ${ }^{(2)}$ \\ ${ }^{1}$ ISEN Brest, Département Vision, L@bISEN, 20 rue Cuirassé Bretagne, CS 42807, 29228 Brest \\ Cedex 2, France. E-mail: ayman.al-falou@isen.fr \\ ${ }^{2}$ Department of Electrical and Computer Engineering, EEB 75, University of South Alabama \\ 6001 USA South Dr. Mobile, AL 36688-0002
}

\begin{abstract}
:
In this paper, we propose a new technique for rotation invariant recognition and tracking of the face of a target person in a given scene. We propose an optimized method for face tracking based on the Fringe-adjusted JTC architecture. To validate our approach, we used the PHPID data base containing faces with various in-plane rotations. To enhance the robustness of the proposed method, we used a three-step optimization technique: (1) utilized the fringe-adjusted filter ( $\mathrm{H}_{\mathrm{FAF}}$ ) in the Fourier plane, $(2)$ added nonlinearity in the Fourier plane after applying the $\mathrm{H}_{\mathrm{FAF}}$ filter, and (3) used a new decision criterion in the correlation plane by considering the correlation peak energy and five largest peaks outside the highest correlation peak. Several tests were made to reduce the number of reference images needed for fast tracking while ensuring robust discrimination and efficient of the desired target.
\end{abstract}

Keywords: correlation, Nonlinear Fringe-adjusted JTC, Face recognition.

\section{Introduction}

Correlation is a robust and discriminative pattern recognition technique widely studied in the literature [1]. Its principle consists in measuring the Degree of similarity between a target image (image to be recognized) and a reference image (belonging to a database). Two families of architectures are used by most researchers to implement the correlation technique: (1) the VanderLugt Correlator (VLC) [2] and (2) the Joint Transform Correlator [3].

In this paper, we consider only the second "JTC" correlation architecture for our proposed Face tracking system. This system must be robust to changes in in-plane rotation of the face and be discriminative. Our objective is to track a subject in a given scene. Before discussing our system, we recall the basic principle of JTC architecture. To enhance the performances of the proposed tracking system (robustness and discrimination) we use an optimized version of this architecture, namely the Fringe-adjusted JTC [4]. In addition, nonlinearity is introduced in the Fourier plane of the JTC correlator. Special attention is given 
to establishing an optimized correlation decision criterion: an adapted version of the PCE (Peak-to-Correlation Energy) is proposed and validated.

\section{Analytical Modeling}

JTC correlation architecture is described in detail in the literature, but we briefly recall the principle of JTC architecture in this paper. For more information, we invite the reader to consult the many works proposed and validated in this area by several researchers and groups working in this field, such as [1.3-4].

The principle of the Joint transform correlator consists in displaying in the input plane of the correlator both the target image " $i$ " (image to be recognized) and the reference image " $r$ ". This plane can be represented mathematically by function " $f$ " described in Eq. (1). To simplify its mathematical representation, we consider only a shift distance (equal to " $\mathrm{x}_{0}$ ") along the $x$ axis between the target image and the reference image.

$$
f(x, y)=i\left(x+x_{0}, y\right)+r\left(x-x_{0}, y\right)
$$

After an first Fourier transformation of the input plane, we obtain its joint spectrum after which a Fourier transformation of the latter gives us the correlation plane (output plane). This plane contains two important items of information: (1) a central autocorrelation peak, and (2) two intercorrelation peaks (the correlation between image " $\mathrm{i}$ " and image reference " $r$ "). These two intercorrelation peaks are located on either side of the central peak at a distance proportional to " $\mathrm{x}_{0}$ ". To optimize this basic architecture Alam et al [4] proposed to introduce a fringe-adjusted filter $\mathrm{H}_{\mathrm{FAF}}$, Eq. (2), in the Fourier plane of the correlator, such that

$$
H_{F A F}(\mu, v)=\frac{B(\mu, v)}{A(\mu, v)+|R(\mu, v)|^{2}}
$$

Where " $R$ " denotes the Fourier transform of the reference considered, " $B$ " is a constant or a function for obtaining a gain greater than unity and " $A$ " is a function for reducing the noise effect. Before discussing the results obtained with this FA_JTC architecture, we present the application considered in this paper. Our application consists in recognizing and tracking a face that changes according to different rotation angles Fig. (1). The system proposed in this paper should allow robust tracking and discrimination for a face turning in different in-plane rotation angles between $-90^{\circ}$ and $90^{\circ}$.

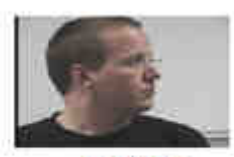

$-90^{\circ}$

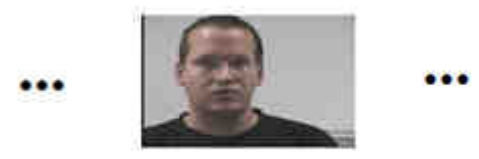

$0^{\circ}$

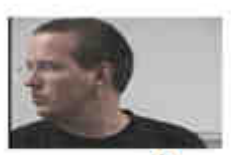

$+90^{\circ}$

Fig. 1. Example of a face (in-plane rotation $\left[-90^{\circ}\right.$ to $\left.\left.+90^{\circ}\right]\right)$. Subject number (1), Base PHPID [6]. 
Figure (2) presents the first results obtained with FA_JTC using a single image reference to construct the filter $\mathrm{H}_{\mathrm{FAF}}$ (the face of the subject presented in Fig. (1) without rotation). The Background is defined as information outside a square around the face in the reference image considered. Then we display in the target image part (half the input plane) the target faces from the database presented in Fig. (1), one after the other in directions -90 ${ }^{\circ}, \ldots, 0^{\circ} \ldots+90^{\circ}$. It should be recalled that the input plane of a JTC is divided into two parts, one reserved for displaying the target image and the other for displaying the references. The aim is to track the face desired from one target image to another.

We initialize the system with a known reference image, i.e. the subject's face (number 1) to be tracked; the position of his face is known. By comparing this reference face with the target image, we obtain two items of information: the presence or not of the subject number (1) in the target image and the position of the subject's face in the target image. Then, only information around the position of the face is selected in this target image. To do this, we multiply the target image with a filter equal to "1" around the position of the face found and "0" elsewhere. Afterwards, we introduce the processed image in the part reserved for references in the FA_JTC input plane. Then a new target image is diplayed, etc..

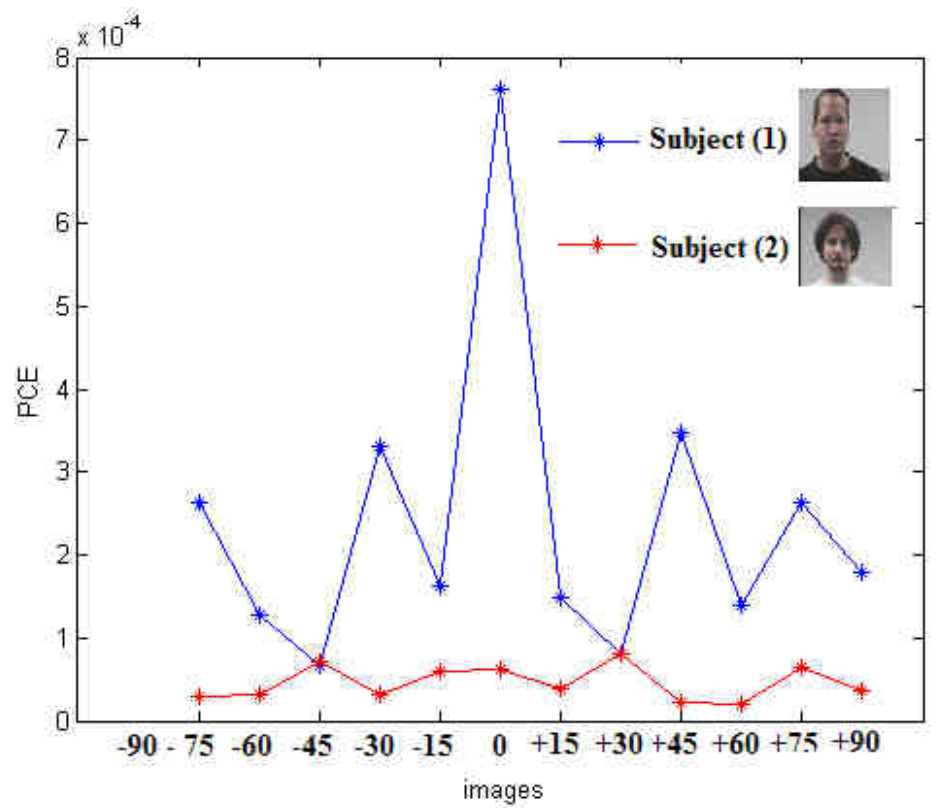

Fig. 2. Depicts results obtained with a classical FJTC using only one reference image position without any rotation in the $\mathrm{H}_{\mathrm{FAF}}$ Filter to define the background noise. We used the PCE criterion

With the various tests performed by using this algorithm, we have identified a tolerance rotation angle equal to $15^{\circ}$ between the target image and reference image in which the correlation remains robust. Beyond this value between the reference and target images, the correlation becomes less robust and may lose track of the subject's face. With a tolerance angle equal to $15^{\circ}$, the tracking problem of a person with in-plane rotation (between -90 and $\left.+90^{\circ}\right)$, consists in correlating "13" couples of images: $(-90,-75)(-75,60)$, $\ldots,(-15,-0),(0,+15) \ldots(+60,+75)(+75,+90)$. The tracking results of subject number $(1)$ are shown in Figure (2) (blue curve). The PCE criterion (Peak-To-Correlation Energy) was used for 
decision-making in the correlation plane [7]. Figure (2) also shows the results when subject number (2) (undesirable subject) is presented in the input plane (False Alarm: red curve). Both curves show that the proposed FA_JTC system gives good results. However, the values of the two curves are too close for a discriminative and robust system.

To optimize the performance of our system, we propose to adapt the function used to manufacture the fringe-adjusted filter $\left(\mathrm{H}_{\mathrm{FAF}}\right)$ [8]. The background noise is calculated for each reference. The results with this first adaptation are shown in Figure (3) which clearly shows that we increase the discrimination of our system, i.e. the difference between the two curves are greater. To further increase the performance of the proposed system, we propose to adapt the decision criterion. Moreover, we propose adding nonlinearity in the Fourier plane of the correlator. These optimizations will be discussed in the next paragraph.

\section{Test Result}

To increase the performance of our system we carried out two other optimizations:

1) We begin this section by proposing an adaptation of the PCE decision criterion used. To do this, we propose to take into account the correlation peak as well as the five highest peaks outside of the area correlation peak. Mathematically, this criterion can be defined by:

Criterion used $=\frac{\text { correlation peak energy }- \text { Average of five highest peaks outside the correlation peak }}{\text { correlation peak energy }}$

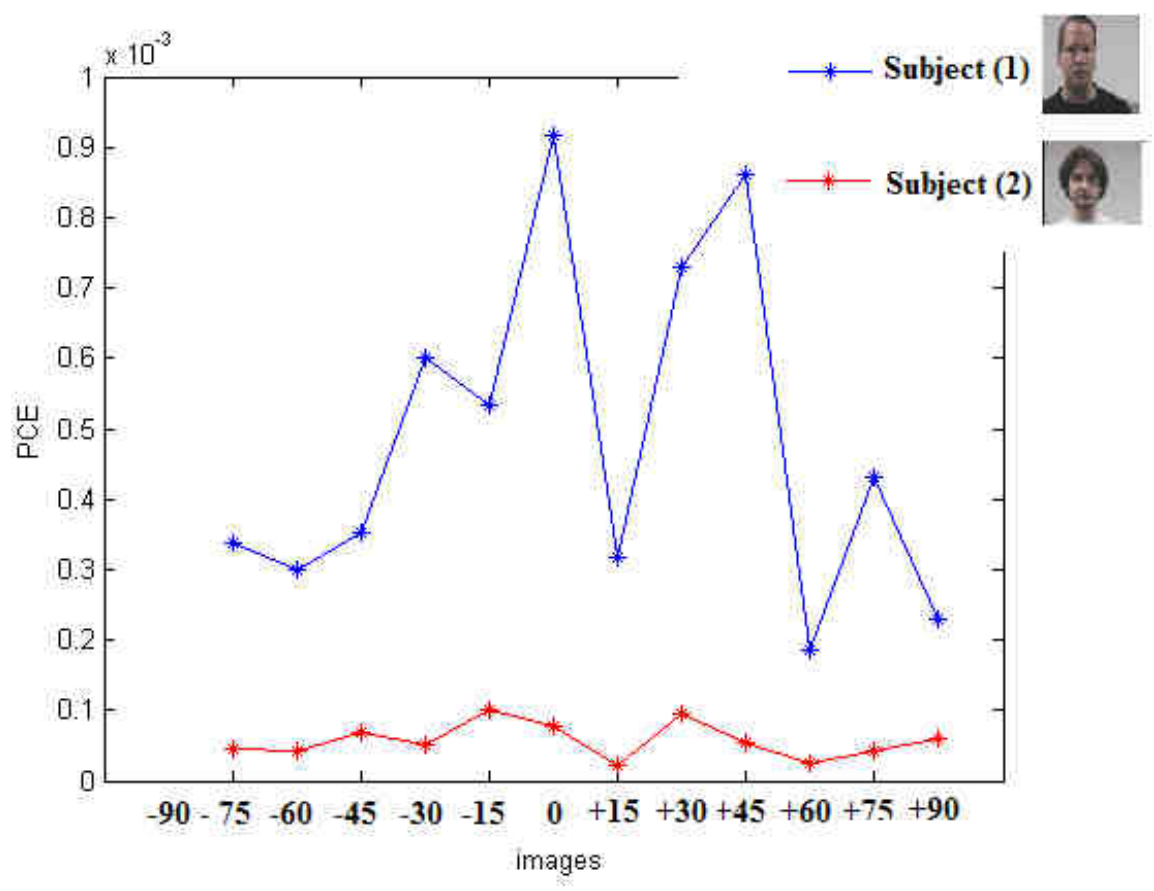

Fig. 3. Results obtained with FJTC; we adapt the $\mathrm{H}_{\mathrm{FAF}}$ filter for each reference image 
2) The second optimization performed in this paragraph is to add non-linearity in the Fourier plane of the correlator [5]: $F_{N L}=F^{k} * \operatorname{sign}(F)$. After performing different tests in our laboratory, we chose $\mathrm{K}=0.75$.

Figure (4) shows the different simulation results obtained with our tracking system based on an optimized FA_JTC correlator. By comparing the two curves presented in Figure (4) with those presented in Figure (1), it can easily be seen that we have succeeded in increasing the discrimination of our system. Indeed the difference between the two curves (red and blue) is larger.

\section{Conclusion}

In this paper we proposed and validated a first version of a robust and discriminative tracking system based on FA_JTC architecture. By choosing a tolerance in-plane rotation angle of $15^{\circ}$ (rotation between the target image and the reference image), we considerably reduced the number of correlations necessary and therefore increased the speed of the system. Moreover, the adaptation of the decision criterion to be used in the output plane, allowed taking into account and minimizing the effect of the false correlation peaks in the correlation plane. This reduced the false alarms. Finally the addition of nonlinearity in the Fourier plane makes it possible to adapt or find a compromise between the robustness and discrimination of our tracking system by changing the value of $\mathrm{K}$.

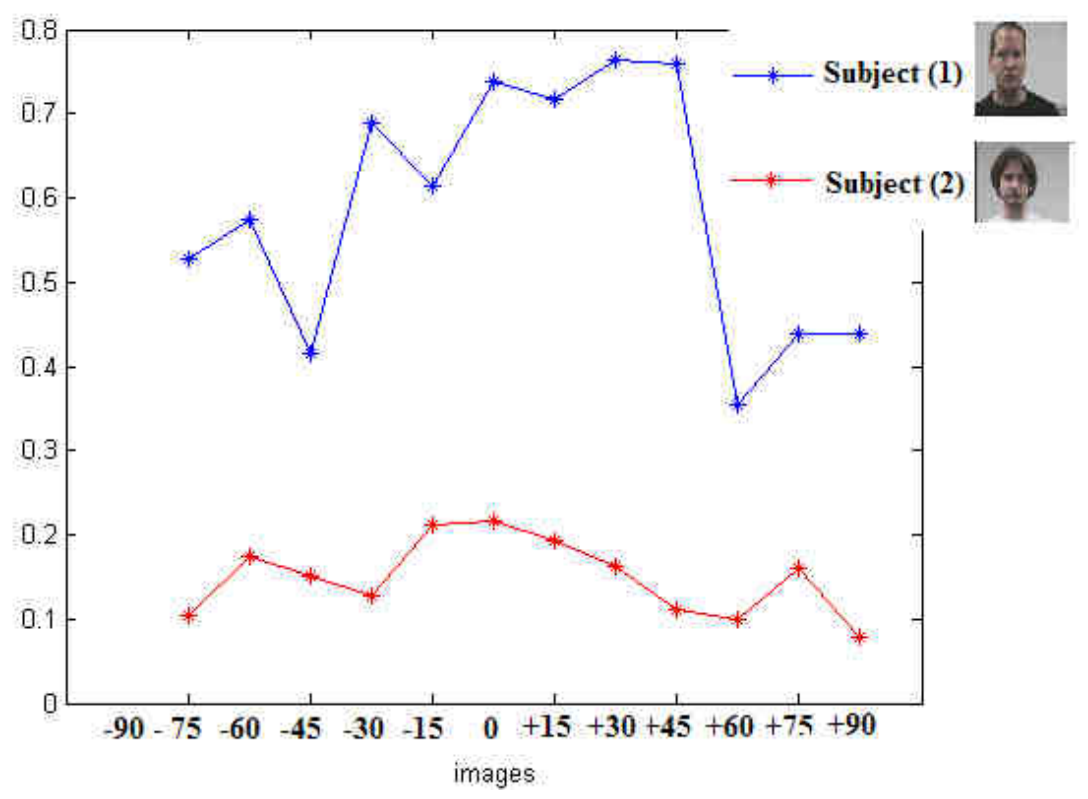

Fig. 4. Results obtained with a non-linearity in the Fourier plane of the FA-JTC and the new criterion

\section{References}

[1] A. Alfalou and C. Brosseau, "Understanding Correlation Techniques for Face Recognition: From Basics to Applications," Face Recognition, Milos Oravec (Ed.), ISBN: 978-953-307-060-5, INTECH. 
[2] A.VanderLugt, "Signal detection by complex spatial filtering," IEEE Trans. Info. Theory, IT10, 139-145 (1964).

[3] C. S. Weaver, J. W. Goodman, "A Technique for Optically Convolving Two Functions," Applied Optics, 5, 1248-1249(1966).

[4] M.S. Alam, M. A. Karim, "Fringe-adjusted joint transform correlation," Applied Optics, 32, 4344-4350 (1993).

[5] J. Bahram, "Nonlinear joint power spectrum based optical correlation," Applied Optics, 28, 2358-2367 (1989).

[6] N. Gourier, D. Hall, and J. L. Crowley, "Estimating Face Orientation from Robust Detection of Salient Facial Features," Proc. of Pointing 2004, ICPR, International Workshop on Visual Observation of Deictic Gestures.

[7] J. L. Horner, "Metrics for assessing pattern-recognition performance," Appl.Opt., 31, 165166. (1992).

[8] M.S. ALAM and A. BAL, "Dynamic target tracking with fringe-adjusted joint transform correlation and template matching," Applied Optics, 43 :4874-4881, 2004. 